Electromagnetic Hot and Dense Nuc

Karl-Heinz Kar

- WA80 Collabo

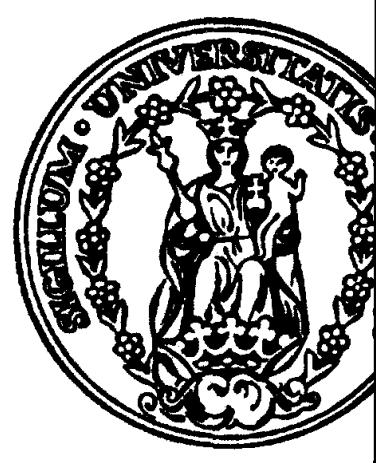

INSTITUT FÜR KE UNIVERSITÄT MI 
IKP-MS-94/0101

\title{
Electromagnetic Probes of Hot and Dense Nuclear Matter
}

\author{
Karl-Heinz Kampert \\ - WA80 Collaboration -
}

T.C. Awes ${ }^{5}$, C. Barlag ${ }^{4}$, F. Berger ${ }^{4}$, M. Bloomer ${ }^{2}$, C. Blume ${ }^{4}$, D. Bock ${ }^{4}$, R. Bock ${ }^{1}$, E.M. Bohne ${ }^{4}$, D. Bucher ${ }^{4}$, A. Claussen ${ }^{4}$, G. Clewing ${ }^{4}$, R. Debbe ${ }^{6}$, A. Eklund ${ }^{3}$, S. Fokin ${ }^{7}$, S. Garpman ${ }^{3}$, R. Glasow ${ }^{4}$, H. $\AA$. Gustafsson $^{3}$, H.H. Gutbrod ${ }^{1}$, O. Hansen ${ }^{6}$, G. Hölker ${ }^{4}$, J. $\mathrm{Idh}^{3}$, M. Ippolitov ${ }^{7}$, P. Jacobs ${ }^{2}$, K.H. Kampert ${ }^{4}$, K. Karadjev ${ }^{7}$, B.W. Kolb ${ }^{1}$, A. Lebedev ${ }^{7}$, H. Löhner ${ }^{8}$, I. Lund ${ }^{8}$, V. Manko ${ }^{7}$, B. Moskowitz ${ }^{6}$, F.E. Obenshain ${ }^{5}$, A. Oskarsson ${ }^{3}$, I. Otterlund ${ }^{3}$, T. Peitzmann ${ }^{4}$, F.Plasil ${ }^{5}$, A.M.Poskanzer ${ }^{2}$, M.Purschke ${ }^{1}$, B. Roters ${ }^{1}$, S.Saini ${ }^{5}$, R.Santo ${ }^{4}$, H.R. Schmidt ${ }^{1}$, K.S $\varnothing$ derstrom $^{3}$, S.P.S $\varnothing r e n s e n^{9}$, K. Steffens ${ }^{4}$, P. Steinhaeuser ${ }^{1}$, E. Stenlund ${ }^{3}$, D. Stüken ${ }^{4}$, A. Vinogradov ${ }^{7}$, H. Wegner ${ }^{6}$, and G.R. Young ${ }^{5}$

1. Gesellschaft für Schwerionenforschung, D-64220 Darmstadt, Fed. Rep. of Germany

2. Lawrence Berkeley Laboratory, Berkeley, California 94720, USA

3. University of Lund, S-22362 Lund, Sweden

4. University of Münster, D-48149 Münster, Fed. Rep. of Germany

5. Oak Ridge National Laboratory, Oak Ridge, Tennessee 37831, USA

6. Brookhaven National Laboratory, Upton, New York 11973, USA

7. Kurchatov Institute of Atomic Energy, Moscow 123182, Russia

8. KVI, University of Groningen, NL-9747 AA Groningen, Netherlands

9. University of Tennessee, Knoxville, Tennessee 37996, USA

Invited talk presented at the NATO Advanced Study Institute on "Hot and Dense Nuclear Matter", Bodrum/Turkey, Sept. 26-Oct. 9, 1993 



\title{
ELECTROMAGNETIC PROBES OF HOT AND DENSE NUCLEAR MATTER
}

\author{
Karl-Heinz Kampert \\ University of Münster \\ Institut für Kernphysik \\ D-48149 Münster \\ and \\ WA80-Collaboration, CERN
}

\section{INTRODUCTION}

The primary motivation for studying high-energy heavy-ion collisions is to investigate nuclear matter under conditions of extremely high densities and temperatures. The response of the nuclear medium to changes of its temperature and density is described by the nuclear matter equation of state which is subject of quantitative discussions in the energy regime of $1 \mathrm{GeV}$ per nucleon. Many new results from experiments adressing these questions at the Berkeley Bevalac and SIS in Darmstadt have been discussed during this meeting. Among the ultimate goals of ultrarelativistic nuclear collisions $\left(E_{\text {proj }} \gtrsim 10 \mathrm{AGeV}\right)$ is the formation and observation of a quark-gluon plasma (QGP) which has been predicted by QCD lattice calculations (for a recent review on this subject the reader is referred to Ref. ${ }^{1}$ ). In such a novel state of matter quarks and gluons are deconfined over an extended volume and chiral symmetry may (partially) be restored. A vigorous experimental programme is now under way at the CERN-SPS and Brookhaven-AGS accelerators with additional preparations being carried out for Brookhaven's Relativistic HeavyIon Collider (RHIC) and CERN's Large Hadron Collider (LHC). The creation and observation of a QGP represents a considerable challenge, both in its experimental realization and also in the theoretical interpretation of the experimental results. The lifetimes involved are of the order of $\approx 10 \mathrm{fm} / \mathrm{c}$ and the detailed dynamics of the collision process may furthermore play an important rôle, because the subsequent hadronization tends to mask the signal from the QGP phase, thus complicating the 
extraction of a clear signal. Nevertheless, much progress has been made both in theory and experiment, and even in the absence of a genuine QGP the study of hot and dense hadronic systems is a fascinating subject from which a great deal can be learned ${ }^{2}$.

Electromagnetic probes are very well suited for studies of strongly interacting particles under conditions from free space to nuclear matter at high densities and temperatures. They carry away virtually unaltered information about the reaction since they do not suffer strong interaction rescattering. This results in mean free paths much bigger than typical sizes of the nuclear systems. Since production rates are rapidly increasing functions of temperature and density, electromagnetic signals provide valuable information on the early hot and dense phases of the reaction and should constitute precious aids in the process of analyzing the behavior of a hot QGP. Early theoretical work on this subject can be found in Refs. $3,4,5,6,7,8$. For hadronic particles the situation is quite different, since their abundances and phase space distributions are changed by the collisions as the final matter expands. However, as with any possible experimental signature of the QGP, a great deal of care must go into the calculation of a corresponding "purely hadronic" signal that is a contribution to the same experimental observables from sources other than a deconfined chiral-symmetric phase. Thus, one must learn if contributions from sources other than the QGP either are dominant in some part of phase space or have so distinct properties so that they can be separated.

To establish an overall picture of the electromagnetic emission, we shall first consider the basic production processes of real and virtual photons in a QGP as compared to a hadron gas and perform rate calculations for static systems at different temperatures. More interestingly for heavy-ion collisions, however, are absolute cross sections. They allow to judge, whether such probes are observable above background in a real experiment. We shall assess the various yields in a rather simple framework of ideal gases by assuming entropy conservation and a one-dimensional Bjørken expansion. Preliminary experimental data from WA80 on direct photon production will be presented and possible interpretations be discussed. Finally, an outlook to future experiments at collider energies will be given.

\section{SOURCES OF REAL AND VIRTUAL PHOTONS}

A system formed during relativistic heavy ion collisions consists of many charged objects moving in close proximity and thus emitting radiation in form of real and virtual photons with the latter being observed as lepton-pairs $\left(\mathrm{e}^{+} \mathrm{e}^{-}\right.$or $\left.\mu^{+} \mu^{-}\right)$. In a QGP those charged objects are represented by (anti-)quarks, whereas in a hadron gas they are represented by mesons and baryons. Photons and lepton-pairs are thus emitted during the hole collision process. This is schematically depicted in Fig. 1 where the invariant mass spectrum of lepton pairs is shown for high energy nuclear collisions.

When the nuclei start to interpenetrate at very high collision energy, their partons will interact non-thermally. In case of real photons the primary production processes (order $\alpha \alpha_{s}$ ) are quark-gluon Compton scattering, $q g \rightarrow q \gamma$, and quarkantiquark annihilation $q \bar{q} \rightarrow g \gamma$ (Fig. 2a, b, respectively). The particular primary process (order $\alpha^{2}$ ) of lepton-pair production is the Drell-Yan process of quark- 


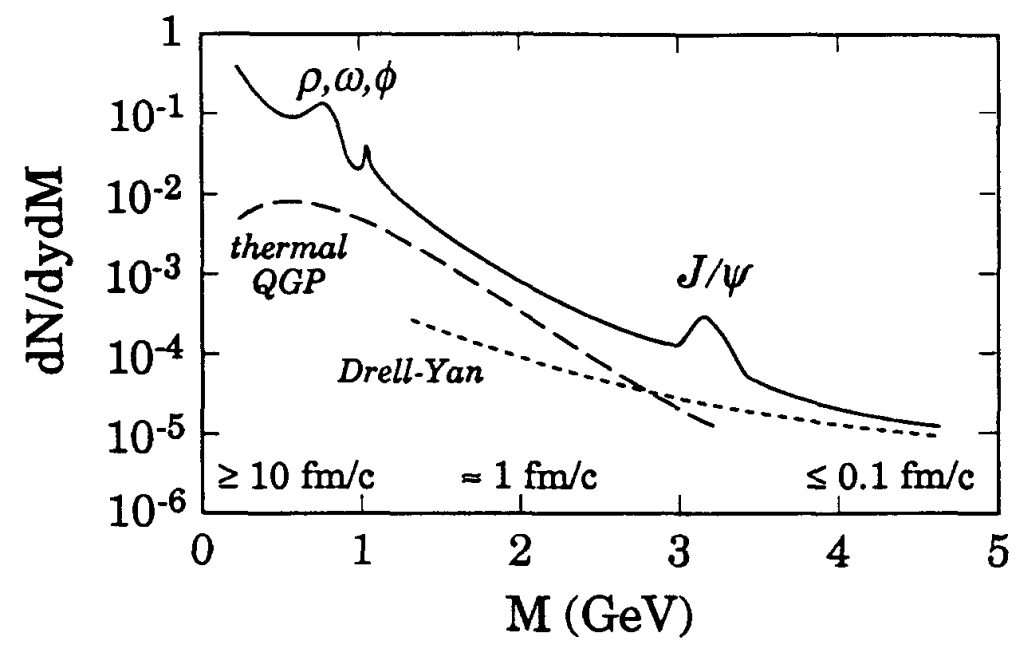

Figure 1. A schematic presentation of the di-lepton mass distribution in high energy nuclear collisions. Typical time scales of their emission are indicated.

antiquark annihilation; $q \bar{q} \rightarrow \gamma^{\star} \rightarrow \ell^{+} \ell^{-}$(Fig. 2c). All of these hard processes are rather well understood and can be treated as interactions of point-like particles in the framework of perturbative QCD. Its comparison to experimental data has provided a great deal of information about the quark- and gluon structure functions $9,10,11$ of nucleons and nuclei and furthermore serves as a reference for the study of the suppression of the $J / \psi$ and higher charmonium and bottonium states in a QGP. As sketched in Fig. 1, lepton-pair spectroscopy allows to identify the decay of various vector mesons, like $J / \psi, \phi, \rho$, and $\omega$ and to study the production properties of these particles. We will return to this important aspect below.

As mentioned above, the differential cross sections of the diagrams in Fig. 2 are well understood and can after carrying out color-sums and spin-averages for the Compton and annihilation graph be expressed as follows ${ }^{12}$.

$$
\frac{d \sigma}{d t}(q g \rightarrow \gamma q)=\pi \alpha \alpha_{s} e_{q}^{2} \frac{1}{6} \frac{-2}{\hat{s}^{2}}\left(\frac{\hat{u}}{\hat{s}}+\frac{\hat{s}}{\hat{u}}\right)
$$

and

$$
\frac{d \sigma}{d t}(q \bar{q} \rightarrow \gamma g)=\pi \alpha \alpha_{s} 2 e_{q}^{2} \frac{4}{9} \frac{2}{\hat{s}^{2}}\left(\frac{\hat{u}}{\hat{t}}+\frac{\hat{t}}{\hat{u}}\right)
$$

with the quark charge $e_{q}$ and the usual Mandelstam invariants $\hat{s}, \hat{t}$ and $\hat{u}$ for the constituent subprocess $\left(\hat{s}=x_{a} x_{b} s\right)$.

\section{Thermal photon radiation from a QGP}

The thermal component in Fig. 1 arises from collisions among quanta which overlap after the primary collision and have a momentum spread characterized by the temperature. Thus, to calculate rates of thermal photon and lepton-pairs in 


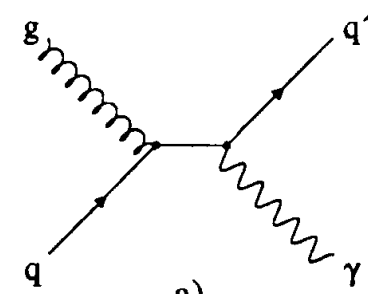

a)

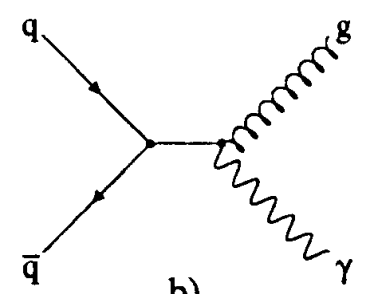

b)

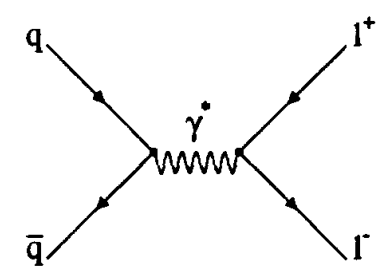

c)

Figure 2. QCD Feynman diagrams of leading order real and virtual photon production in a QGP; (a) quark-gluon Compton $q g \rightarrow q \gamma$, (b) quark-antiquark annihilation $q \bar{q} \rightarrow g \gamma$, (c) lepton pair production by quark-antiquark annihilation $q \bar{q} \rightarrow \gamma^{\star} \rightarrow \ell^{+} \ell^{-}$.

a QGP one assumes Fermi-Dirac and Bose-Einstein distributions of a certain temperature for the quarks and gluons, respectively. Sometimes, both distributions are approximated for simplicity by a Maxwell-Boltzmann distribution ${ }^{13,14}$. Perturbative QCD at finite temperature suffers, however, from serious problems leading to infrared singularities and gauge dependence for many observables calculated in this way. The reason for this is the fact that naive perturbation theory of finite temperature is incomplete, i.e. an expansion in the number of loops is not equivalent to an expansion in the coupling constant $g$. This problem can be circumvented by using effective propagators and vertices based on a resummation of the so-called hard thermal loop diagrams, as was shown by Braaten and Pisarki ${ }^{15}$. In this way, medium effects, e.g. Debye screening, are included and improve the infrared behavior of the results drastically. At the same time, effective perturbation theory leads to consistent results for observables, i.e. gauge independent results which are complete to leading order in $g$.

The resummation technique has been applied by Baier et al. ${ }^{16}$ and Ruuskanen 14 In both cases the thermal invariant emission rate of photons is related to the imaginary part of the photon self-energy 17,7 . For real photons the consistent lowest order (Fig. 2a, b) calculation of emission rate at fixed temperature $T$ can then be written as 16

$$
E \frac{\mathrm{d} R_{\gamma}}{\mathrm{d}^{3} p \mathrm{~d}^{4} x} \cong\left(\sum Q_{f}^{2}\right) \frac{\alpha \alpha_{s}}{2 \pi^{2}} T^{2} e^{-\frac{E}{T}} \ln \left(\frac{c}{\alpha_{s}} \frac{E}{T}\right)
$$

with the constant $c \cong 0.23$ and where the bare quark mass has already been replaced by the thermal mass $m_{\beta}=\frac{2 \pi}{3} \alpha_{s} T^{2}$. A slightly different result is obtained by Ruuskanen ${ }^{14}$, mainly because of the approximation of the thermal distributions by a Boltzmann law. A quantitative comparison ${ }^{18}$ shows that the rate at a temperature of $T=200 \mathrm{MeV} / \mathrm{c}$ is thereby enhanced compared to Baier et al. ${ }^{16}$ by 


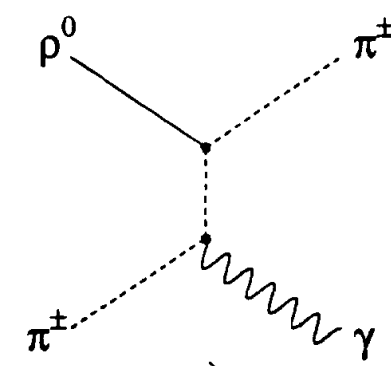

a)

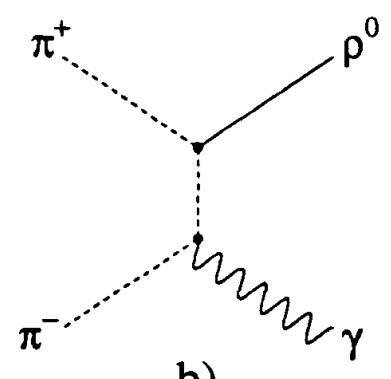

b)

Figure 3. Feynman diagrams of photon production in a hadron gas; (a) $\pi \rho$ Compton with an intermediate virtual $\pi, \rho$, or $A_{1}(1260)$ meson, (b) $\pi^{+} \pi^{-}$annihilation.

approximately a factor of two. Almost identical emission rates to Baier's calculations, on the other hand, were found by Hwa and Kajantie ${ }^{8}$, who have evaluated the Feynman graphs in an effective manner by an approximation to high energy $\mathrm{e}^{+} \mathrm{e}^{-}$data.

\section{Thermal photon radiation from a Hadron-Gas}

Only recently it has been pointed out by Kapusta et al. ${ }^{19}$ that a hot hadronic gas radiates thermal photons with similar emission rates as a QGP of the same temperature. The dominant contribution comes from the reactions $\pi \pi \rightarrow \rho \gamma$ and $\pi \rho \rightarrow \rho \gamma$ (Fig. 3). Xiong et al. ${ }^{20}$ later pointed out that the latter of the two processes proceeds in a hot hadron gas not only via an intermediate virtual $\pi$ or $\rho$, but also via the broad $A_{1}(1260)$ resonance.

If form-factors are neglected, i.e. if particles are treated again as point-like objects, the evaluation of the Feynman graphs proceeds in an almost identical fashion as sketched above for the diagrams of Fig. 2. A quantitative comparison of the different contributions shows that for a thermal hadron gas of $T=150 \mathrm{MeV} / \mathrm{c}$ the pion annihilation dominates for $p_{\perp} \lesssim 0.7 \mathrm{GeV} / \mathrm{c}$, while the $\pi \rho$ channel dominates for large $p_{\perp}$. Here, the photon production via the intermediate $A_{1}$ amounts to approximately twice the yield compared to the intermediate $\pi$ and $\rho$ state.

Future calculations should take into account also form factors. A rough estimate shows that this will reduce the photon rate at $p_{\perp} \approx 2.5 \mathrm{GeV} / \mathrm{c}$ by a factor of approximately $2.8^{19}$.

\section{Lepton-Pair Spectroscopy of a Hadron-Gas}

Lepton-pair emission is, similar to that of photons, not only expected for a thermalized QGP but also for a thermal hadron gas. As discussed above, the electromagnetic radiation couples to hadronic (mesonic) matter via vector mesons. These vector mesons carry the same quantum numbers as the photon and are dominated by the $\rho, \omega$, and $\phi$. They decay with a branching ratio of $\sim 10^{-4}$ into $\mathrm{e}^{+} \mathrm{e}^{-}$or $\mu^{+} \mu^{-}$pairs. This picture is well known as the vector dominance model (VDM) and provides a means to calculate by the aid of experimental form factors the expected rates. 
The lifetime of the $\rho$-meson of approximately $1 \mathrm{fm} / \mathrm{c}$ combined with the decay into lepton pairs opens an exciting diagnostics tool for hot and dense nuclear matter. Because the lifetime is very short compared to typical time-scales of the collision, $\rho$ mesons decay almost instantaneously after their formation in the interior of the hot and dense matter. Since lepton pairs will escape almost unaltered by the surrounding matter their spectroscopy allows to test the properties of those particles inside the medium. Changes of the width and mass may be expected according to the density and temperature of the system 21,22 . Of course, such a diagnostics tool is not provided by the dominant $\pi \pi$-decay channel, since those pions will strongly be affected by rescattering before reaching the detector.

An experiment, however, has to cope with several competing sources of lepton pairs. At high mass this is the perturbatively treated Drell-Yan process. However, the mass region $M \lessgtr 1 \mathrm{GeV}$ constitutes the major part of the measured yield (cf. Fig. 1), but the present knowledge and understanding of the intermediate and low mass data in hadronic collisions is rather limited. Recent studies $23,24,22$ show that one has to consider bremsstrahlung contributions from $N N, N N \pi, N N \pi \pi$, etc., radiative decays of the $\Delta$-resonance, direct $\pi^{+} \pi^{-}$annihilation, and Dalitz decays of the $\eta$ and $\pi^{0}$ in the $\mathrm{e}^{+} \mathrm{e}^{-}$channel and Dalitz decays of the $\omega$ and $\eta$ in the $\mu^{+} \mu^{-}$channel. The relative importance of each of these channels depends furthermore on the global conditions of the system, i.e. on the projectile energy, the mass of the fireball, its density, temperature, etc., so that precise predictions seem hard to be achieved at present. However, such a knowledge is mandatory for any firm conclusions on thermal lepton-pair production.

\section{EXPECTED YIELDS IN NUCLEAR COLLISIONS}

Up to now, only the photon and di-lepton radiation of static systems with fixed temperature was discussed. Nuclear collisions are, however, highly dynamic systems where even the question about a thermal equilibrium may be raised. Based on results from kinetic theories and experimental data, which provide at least some evidence for a local equilibrium, we will in the following proceed with the assumption of a thermalized system and calculate in a rather idealized way the real photon production in nuclear collisions. We shall assume that the system starts with a QGP of temperature $T_{i}$ being formed after some initial thermalization time $\tau_{i}$. Then it cools isentropically due to longitudinal Bjørken expansion. Furthermore, we assume a first order phase transition to a mesonic gas at temperature $T_{c} \cong 160 \mathrm{MeV} / \mathrm{c}$. The calculation is stopped after the hadron gas has cooled to a freeze-out temperature of $T_{f} \approx 100 \mathrm{MeV} / \mathrm{c}$. Figure 4 visualizes the time evolution and its relevant parameters.

The photon yield from the QGP is largely dominated by the initial temperature $T_{i}$ which in turn is linked to the initial time $\tau_{i}$ via the entropy density of the system. The entropy density $\zeta$ can be estimated from the experimental particle multiplicities $d N / d y$ and the projectile radius $\propto A_{p}^{2 / 3}$. Using the uncertainty principle $\tau_{i} Z \hbar /\langle E\rangle$, we may write (compare Ref. 25,26 )

$$
\tau_{i} \approx 0.93 \sqrt{\frac{A_{p}^{2 / 3}}{d N / d y}}(\mathrm{fm} / \mathrm{c})
$$




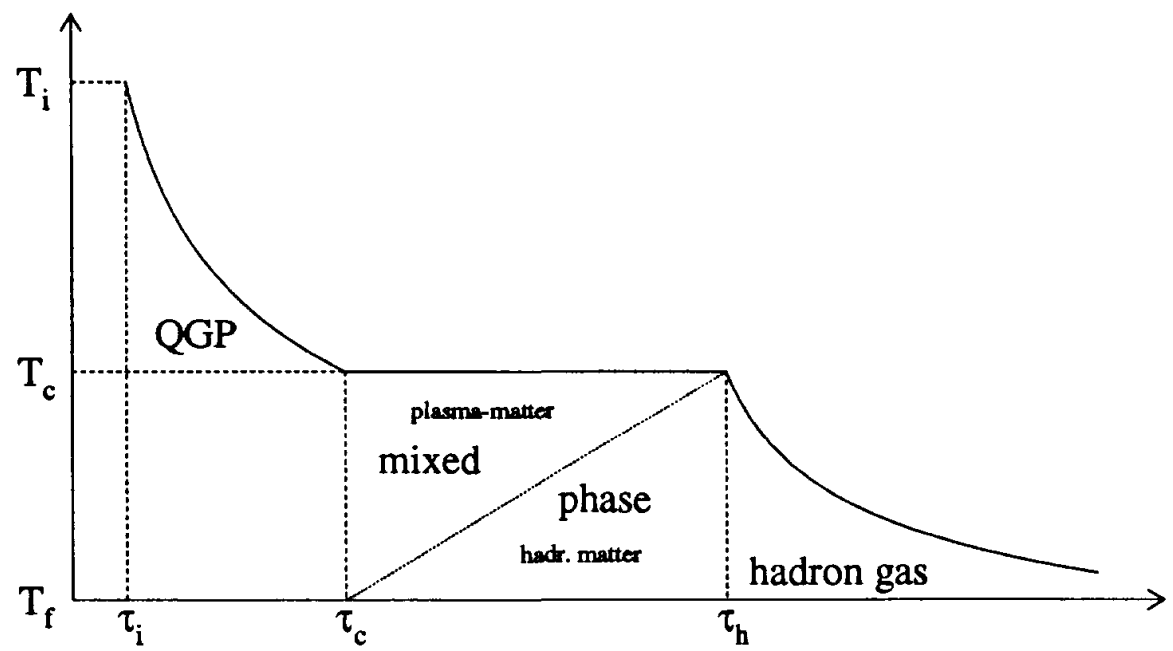

Figure 4. Time evolution of the temperature in a nuclear reaction with a first order phase transition.

which represents a lower limit for the initial time. Now we can use this relation and estimate the initial temperature from the entropy density of an ideal gas ${ }^{8}$, $\zeta=d \varepsilon / d T=4 a T^{3}(a=$ degrees of freedom $)$ and find

$$
T_{i} \approx 81 \sqrt{\frac{d N / d y}{A_{p}^{2 / 3}}}(\mathrm{MeV} / \mathrm{c}) .
$$

Entropy conservation, $\zeta_{i} \tau_{i}=$ const $=\zeta \tau$, and the ideal gas equation of state $P=\varepsilon / 3$ then determines the cooling rate $T(\tau)=T_{i} \cdot\left(\tau_{i} / \tau\right)^{1 / 3}$ until the critical temperature is reached at time $\tau_{c}=\tau_{i} \cdot\left(T_{i} / T_{c}\right)^{3}$. Now the mixed phase starts and plasma matter is converted into hadronic matter. Entropy conservation still holds and the end of the mixed phase is then given by $\tau_{h}=r \tau_{c}$ with $r$ being the ratio of the degrees of freedom in the QGP over the hadron gas. In analogy to above, the system then cools again according to $T(\tau)=T_{c} \cdot\left(\tau_{h} / \tau\right)^{1 / 3}$ until the end of the reaction is marked by the freeze-out temperature $T_{f}$.

The total yield is obtained by the time integration over the three phases. The initial temperature for central $\mathrm{S}+\mathrm{Au}$ collisions has been assumed $T_{i}=336 \mathrm{MeV} / \mathrm{c}$ and $228 \mathrm{MeV} / \mathrm{c}$, both of which are lower than expected from eqn. 5 . The number of degrees of freedom was assumed to be $r=42.25 / 6.6$ in order to take into account contributions from quarks heavier than the $u$ and $d$ and mesons heavier than the $\pi$.

Experimentally, the relevant quantity is the $\gamma / \pi^{0}$-ratio rather than the cross section of thermal photons alone. This is because an experiment has to cope with a large background of photons from the $\pi^{0}$ decay. The $\gamma / \pi^{0}$-ratio thus represents a measure of the surplus of single gammas compared to gammas from meson decays and tells, whether the amount of single photons is sufficient to be detected by an experiment. Using the experimental $\pi^{0}$-spectra, the $\gamma_{\text {thermal }} / \pi^{0}$ can now be calculated and its result is presented in Fig. 5 for central $\mathrm{S}+\mathrm{Au}$ reactions ${ }^{18}$. According to these calculations, photons from a hadron gas dominate the region $p_{\perp} \lesssim 2.5 \mathrm{GeV} / \mathrm{c}$. The spectral slope, $d \sigma / d p_{\perp}\left(\gamma_{\mathrm{HG}}\right)$, reflects in this scenario basically the temperature 


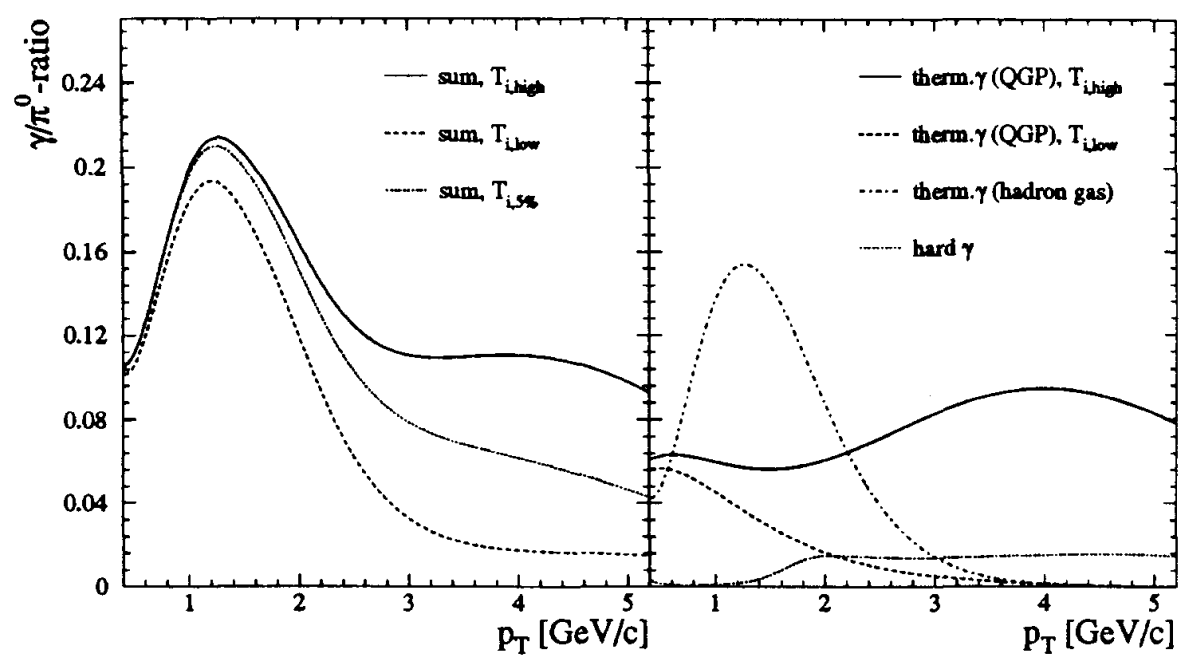

Figure 5. Calculated $\gamma / \pi^{0}$-ratio for central $\mathrm{S}+\mathrm{Au}$ collisions at $200 \mathrm{AGeV}$. The left panel shows the total yield and the right panel its differential contributions. The three different initial temperatures are $T_{i}=338,310$, and $228 \mathrm{MeV} / \mathrm{c}^{18}$

of the mixed phase. Photons from the QGP exhibit the expected strong variation with the initial temperature. For the lowest of the three temperatures, they would remain unobserved within the present experimental uncertainty, but would be clearly visible for the upper temperature. The figure also shows that hard direct photons from the initial state of the collision do not play a significant rôle at SPS fixed target energies.

Finally, it should be stressed again that there are certain simplifications in these calculations; ( $i$ ) thermal equilibria are assumed, something which should be checked and verified by parton kinetic simulations 27 , (ii) the rate calculations were performed for zero chemical potentials $\mu_{q} \cong \mu_{B} / 3 \cong 0$ which are unlikely to be reached in present experiments. The inclusion of baryons is expected to reduce the photon yield at fixed energy density ${ }^{28}$. (iii) Scale invariance according to Bjørken expansion is assumed, but not supported by present experiments. (iv) Transverse expansion effects are neglected and would tend to reduce the photon yield in the late phase of the reaction, i.e. in the hadron gas. Obviously, there are large uncertainties in such calculations and the results should only be regarded a rough estimate. Much more work is still needed and many theoretical efforts are underway particularly at collider energies. Independent of the quantitative results of such calculations experiments may investigate scaling properties of single photons to learn about its origin. The most important characteristics is the expected scaling with $(d N / d y)^{2}$, since quark-antiquark and quark-gluon collisions produce the photons.

\section{EXPERIMENTAL MEASUREMENTS}

Summarizing the results from the previous two sections, thermal photons and lepton-pairs are both considered unique probes of a quark gluon plasma and hot 
hadronic gas. Lepton-pair experiments require hadron-blind detection techniques in order to suppress signals from the $\sim 10^{4}$ more abundantly produced hadrons. In $\mu^{+} \mu^{-}$experiments this is usually achieved by massive absorber materials behind the target so that only muons can penetrate and be detected in wire chambers. Their momenta are usually measured by their curvature in a magnetic field. Measurements of $\mathrm{e}^{+} \mathrm{e}^{-}$-pairs require detectors inherently blind to hadrons. An example of such kind of experiment is NA45 (CERES) at CERN ${ }^{29}$. It employs ring imaging Cherenkov detectors (RICH) with a radiator such that only electrons and very high energetic pions exceed the Cherenkov threshold of of $\gamma_{\text {th }} \cong 32$. Such experiments have succeeded to identity vector mesons, but the questions of thermal lepton-pair radiation was not yet addressed.

Single photon radiation is studied experimentally by two different approaches, called the 'direct' and 'conversion' method. The former one uses a thin converter behind the target (with a thickness of some percent of a radiation length, which may be the target itself) to convert a small amount of photons into $\mathrm{e}^{+} \mathrm{e}^{-}$-pairs. These may then be investigated by a lepton pair experiment as discussed above. An example of such an experiment is again NA45. The direct method, on the other hand, employs finely granulated electromagnetic calorimeters to measure the photons themselves. The calorimeter is chosen in such a way that $\pi^{0}$ - and $\eta$-mesons can be identified by their two-photon decay. The important advantage the direct method over all of the aforementioned techniques is that the background of the thermal radiation is measured within the same experiment so that there is no need to rely on (imprecise) calculations. Such kind of technique is employed by the WA80 and WA93 experiments at CERN. The following discussion will concentrate on data from this experiment. Results from the photon conversion experiment NA45 have been presented by Specht ${ }^{29}$ on this conference.

\section{Results from WA80}

In order to allow for the subtraction of photons from neutral meson decays measured within the same experiment, the detector is primarily designed for high precision $\pi^{0}$ and $\eta$ reconstruction 30,31 . This is achieved by a 3,800 modules leadglass calorimeter covering the pseudorapidity range $2.1 \leq \eta \leq 2.9$ at a distance of $9 \mathrm{~m}$ to the target. To allow tagging of charged particles, a 40,000 pad streamertube detector is placed in front of the lead-glass. With that detector the accessible $p_{\perp}$-range in $200 \mathrm{AGeV} \mathrm{S}+\mathrm{Au}$ reactions is $0.2 \leq p_{\perp} \leq 4.5 \mathrm{GeV} / \mathrm{c}$ for $\pi^{0}$-mesons and $0.5 \leq p_{\perp} \leq 2.5 \mathrm{GeV} / \mathrm{c}$ for $\eta$-mesons. The experimental trigger allows selection of different ranges of centrality to allow for comparisons of spectra from central and peripheral events.

Among the most important experimental difficulties in measuring single photons in high energy nuclear collisions is the precise determination of the photon and $\pi^{0}$ reconstruction efficiencies, $\varepsilon_{\gamma}$ and $\varepsilon_{\pi^{0}}$, respectively. Their calculation is based on the actual experimental data and is performed by superimposing single hadronic and electromagnetic showers on a raw-data level to the measured heavy-ion events. The additional showers are generated either by the GEANT simulation package and assuming phase space distributions of the various particles according to experimental data or are, for reasons of consistency checks, taken from very peripheral $\mathrm{S}+\mathrm{Au}$ reactions. The artificial events are processed with the same chain of shower re- 


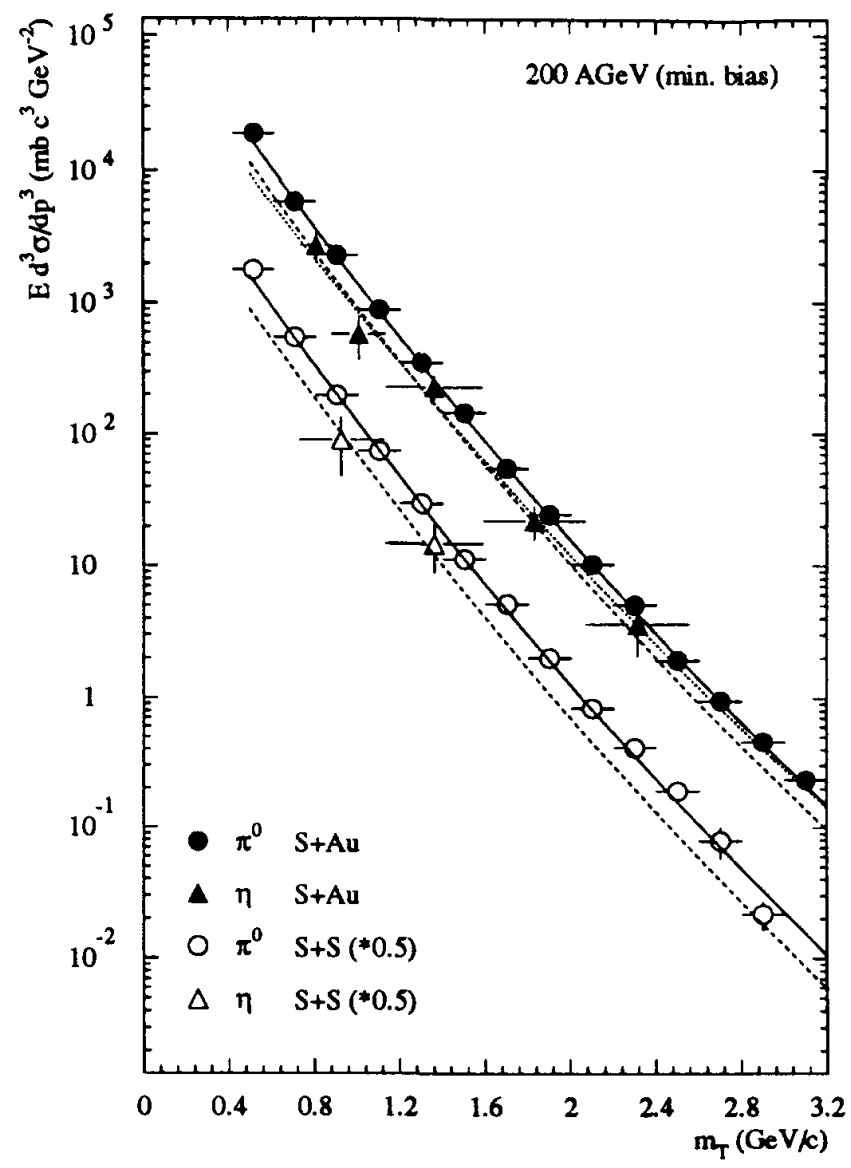

Figure 6. Invariant cross sections of $\pi^{0}$ - and $\eta$-mesons as a function of transverse mass for 200 $\mathrm{AGeV} \mathrm{S}+\mathrm{Au}$ and $\mathrm{S}+\mathrm{S}$ minimum bias data ${ }^{32}$. The $\mathrm{S}+\mathrm{S}$ data are scaled by a factor 0.5 for a better presentation. The different lines represent fits to a function $C \cdot\left[p_{0} /\left(p_{0}+m_{\perp}\right)\right]^{n}$.

construction routines as is used for real data events. The photon reconstruction efficiency is then defined by the ratio of the reconstructed photon spectrum divided by the known input photon spectrum and is found to depend both on the local particle density and on the transverse momentum of the photon. As a typical result for central $\mathrm{S}+\mathrm{Au}$ events, the correction according to $\varepsilon_{\gamma}\left(\varrho_{\text {local }}, p_{\perp},\right)$ leads to a change of the inverse slope parameter in the inclusive photon $p_{\perp}$-distributions from $T_{\text {raw }}=210 \mathrm{MeV}$ to $T_{\text {corr }}=207 \mathrm{MeV}$. The systematic and statistical uncertainty in the photon (and $\pi^{0}$ ) reconstruction efficiency is found to be approximately $3 \%$.

Differential cross sections of $\pi^{0}$ and $\eta$ mesons are obtained by measuring their yields above the combinatorial background in invariant mass distributions of $\gamma \gamma$ pairs selected according to the variable of interest. In order to determine the precise shape (and yield) of the background under the $\pi^{0}(\eta)$ peak, a mixed event method has been developed. Here, the invariant mass distribution is constructed by combining photons from one event with those from another one of the same global characteristics.

Acceptance and efficiency corrected invariant cross sections of $\pi^{0}$ and $\eta$ are shown in Fig. 6 as a function of transverse mass $m_{\perp}=\sqrt{p_{\perp}^{2}+m_{0}}$ for minimum bias, 
$\mathrm{S}+\mathrm{S}$ and $\mathrm{S}+\mathrm{Au}$ reactions. In addition to the statistical errors, systematic errors of the acceptance and efficiency correction are added in quadrature. In such a representation the $\pi^{0}$-mesons of both systems exhibit a concave shaped spectrum with a similar shape observed within the experimental uncertainties for the $\eta$-mesons. The data are thus compatible with the so called phenomenological $m_{T}$-scaling behavior. The $\eta / \pi^{0}$-ratio in bins of equal width in $m_{\perp}$ or for very large transverse momentum is found to be $0.66 \pm 0.09$ and $0.57 \pm 0.18$ for $S+A u$ and $S+S$ data, respectively 32 . A corresponding analysis of $\mathrm{p}+\mathrm{p}, \pi+\mathrm{p}$, and $\mathrm{p}+\mathrm{C}$ reactions found in the literature and taken at $\sqrt{s}=24.3-62 \mathrm{GeV}$ yields $0.55 \pm 0.02$.

The single photon yield is generally expressed in terms of the $\gamma / \pi^{0}$-ratio and can now be extracted from the measured $\gamma, \pi^{0}$, and $\eta$ yields according to the expression 9,33 .

$$
\frac{\gamma}{\pi^{0}}=\frac{N_{\gamma}}{N_{\pi^{0}}} \cdot \frac{\varepsilon_{\pi^{0}}}{\varepsilon_{\gamma}} \cdot A_{\mathrm{geo}}-\left(R_{\pi^{0}}+R_{\eta}+R_{X}\right)
$$

where $\varepsilon_{\pi^{0}}$ and $\varepsilon_{\gamma}$ denote the photon and $\pi^{0}$ reconstruction efficiencies, respectively. $A_{\text {geo }}$ is the geometrical acceptance of the detector for $\pi^{0}$ 's, and $R_{\pi^{0}}, R_{\eta}$, and $R_{X}$ are the Monte-Carlo calculated ratios of observed background photons from measured $\pi^{0}$ and $\eta$ mesons, and higher (non-measured) resonances, respectively. Such a presentation of data has the advantage that certain experimental errors, e.g. the absolute cross section normalization, cancel out thus allowing a total experimental sensitive in the $\gamma / \pi^{0}$ ratio of up to $5 \%$.

In case of high heavy-ion reactions where the $\pi^{0}$ and $\eta$ production cross sections are in principle unknown it is very important to measure those data in the same experiment and in the appropriate $p_{\perp}$ region. Heavier resonances are then to a large part automatically taken already into account, since the major fraction of these resonances decays via the $\pi^{0}$ or $\eta$ branch (e.g. $\eta^{\prime} \rightarrow \pi^{0} \pi^{0} \eta$ ) and is therefore already contained in the measured spectra. The different hadronic photon contributions as calculated from the experimental $\pi^{0}$ and $\eta$ yields and complemented by assumed relative production ratios of heavier particles are displayed in Fig. 7 as a function of $p_{\perp}$. Besides the $\pi^{0}$ decay the most important hadronic photon background is the $\eta \rightarrow \gamma \gamma$ decay. These photons amount to approximately $10 \%$ compared to those from the $\pi^{0}$ while the sum of all heavier resonances contributes on the 1-2\% level. Possible inherent systematic uncertainties when assuming their production cross-sections from different experiments and reactions are thus suppressed by approximately the same factor.

The single photon yield, obtained after subtracting the calculated decay contributions from Fig. 7, is shown in Fig. 8 for central $\mathrm{S}+\mathrm{Au}$ reactions. Different than in the peripheral data the central sample shows a positive signal and a slight increase towards low $p_{\perp}$. The error bars represent the sum of all collected statistical and systematic uncertainties. Assuming a gaussian distribution, we arrive at an uncertainty of $7-8 \%$ and $8-15 \%$ for $p_{\perp}$ bins below and above $2 \mathrm{GeV} / \mathrm{c}$, respectively. The excess of photons in central data over the expected yield from the hadronic background for $p_{\perp} \leqslant 2 \mathrm{GeV} / \mathrm{c}$ thus reaches a level of approximately 2 standard deviations in each point. Data from peripheral collisions are on the other hand well described by known hadronic sources. To allow for comparisons with other experiments and to demonstrate the variation of the 


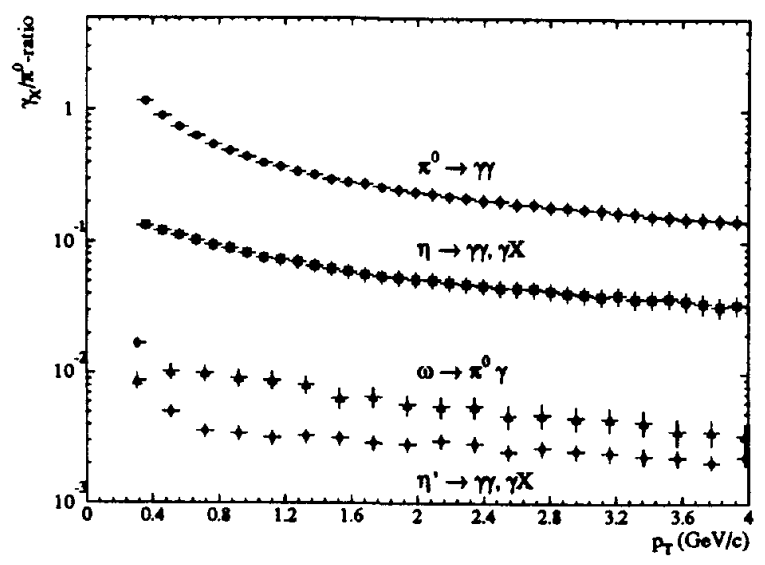

Figure 7. Different hadronic photon contributions normalized to the $\pi^{0}$ yield as a function of $p_{\perp}$ for central $\mathrm{S}+\mathrm{Au}$ reactions.

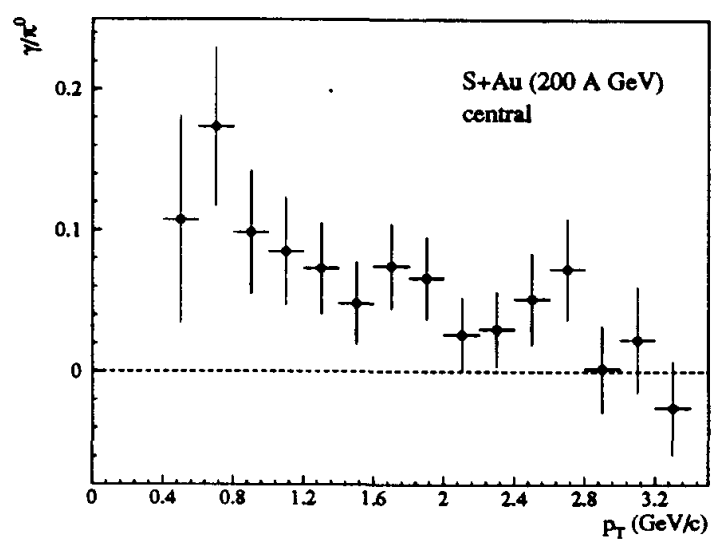

Figure 8. Preliminary background subtracted $\gamma / \pi^{0}$ ratio for central $200 \mathrm{AGeV} \mathrm{S}+\mathrm{Au}$ reactions as a function of $p_{\perp}$.

signal between central and peripheral data one may integrate the $\gamma / \pi^{0}$ ratio in $0.4 \leq p_{\perp} \leq 2.0 \mathrm{GeV} / \mathrm{c}$ to find $\gamma /\left.\pi^{0}\right|_{\text {int }} ^{\text {cen }}=0.091 \pm 0.016$ (stat) \pm 0.058 (syst) in central events and $\gamma /\left.\pi^{0}\right|_{\text {int }} ^{\text {per }}=0.013 \pm 0.019$ (stat) \pm 0.058 (syst). NA45 $5^{29}$ finds for central collisions of the same reaction $\gamma / \pi^{0}=0.075 \pm 0.005$ (stat) \pm 0.110 (syst) and $\mathrm{NA} 34^{34}$ reports no evidence for an excess within an experimental uncertainty of $\sigma\left(\gamma / \pi^{0}\right)=(0.04-0.11)$ (stat) \pm 0.09 (sys). All results are thus compatible with each other, but the uncertainty of the latter two experiments is still too large to allow for a sensitive comparison.

The spectral shape observed in Fig. 8 resembles very much the shape expected from the hadronic interactions shown in Fig. 5. At this stage of the analysis, however, no quantitative comparison to calculated photon spectra will be made. This is because of both large uncertainties in the calculations, as stated above, and the preliminary character of the experimental data. A more obvious link to the temperature of the system may be given instead by the invariant cross section of single 


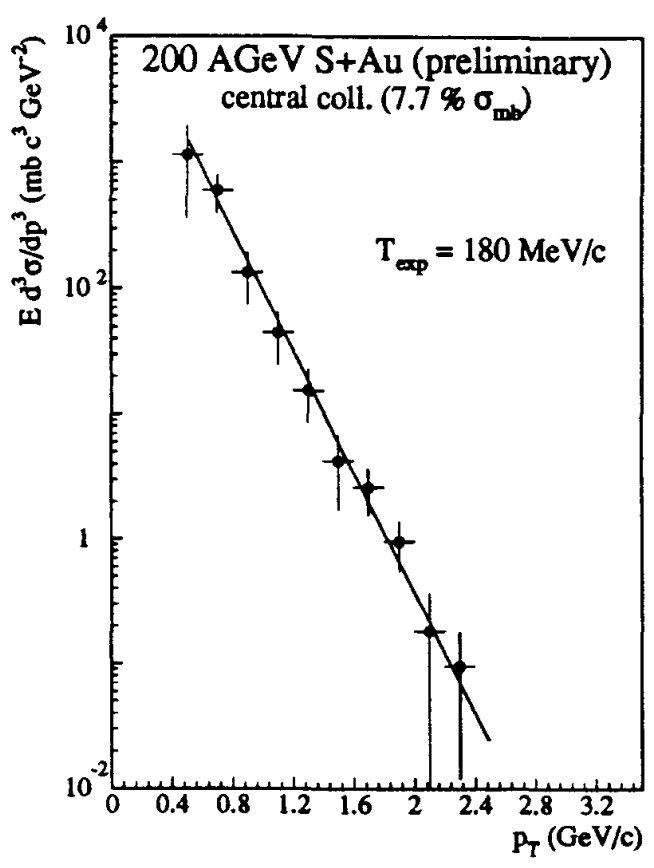

Figure 9. Preliminary invariant cross section of single photons from central S + Au data (WA80). The line shows an exponential distribution of $T_{\exp }=180 \mathrm{MeV} / \mathrm{c}$.

photons which is obtained from Fig. 8 by multiplying with the experimental $\pi^{0}$ distribution. Such a preliminary distribution is shown in Fig. 9. Fitting an exponential distribution to the data points yields a slope parameter of $T_{\exp } \cong 180 \mathrm{MeV} / \mathrm{c}$ which would - after taking into account the decay kinematics of the Feynman diagrams of Fig. 3 - translate into a hadronic gas temperature of $T_{\mathrm{HG}} \approx 165 \mathrm{MeV} / \mathrm{c}$.

Before any firm conclusions can be drawn from the data, more work is still needed to verify the significance of the photon excess. The most time consuming and critical work is the Monte-Carlo procedure to determine the $\gamma, \pi^{0}$, and $\eta$ reconstruction efficiencies. Therefore, an independent re-analysis is planned in order to gain extra confidence in the estimated systematic errors.

\section{SUMMARY AND CONCLUSIONS}

The measurement of photons and lepton-pairs provides a powerful tool to learn about the properties of hot and dense matter over a wide range of bombarding energy. Informations extracted from these signals are to a large part complementary with some common features. An important and unique aspect of lepton-pair spectroscopy is the possibility to infer properties of the excited medium from modifications observed in the leptonic decay-width and mass of vector mesons. After pioneering work on this subject performed by the DLS-Collaboration ${ }^{35}$ is being terminated due to the recent shutdown of the Bevalac, a challenging experimental program is now under discussion by the HADES-Collaboration at GSI. At CERN- 
SPS energies the thermal 'black-body radiation' of a hot fireball is of primary interest, because it provides means to measure its temperature in the early phase of the collision. High temperatures, i.e. large photon and di-lepton yields, combined with high entropy densities are expected for a formation of a quark-gluon plasma. From a theoretical point of view, both of these probes are equally well suited. However, experimentally real photons measured by the 'direct' detection technique with $\pi^{0}$ and $\eta$ identification are advantageous, because the hadronic background to the signal can be subtracted based on the experimental data alone without any relevant theoretical assumption. Such an experiment allows for a precision in the $\gamma / \pi^{0}$-ratio of $5-6 \%$ as has been demonstrated by WA80 36,37 .

Preliminary results show for the first time an excess of photons in central nuclear collisions at transverse momenta $p_{\perp} \lesssim 2.5 \mathrm{GeV} / \mathrm{c}$. Calculations of single photons from various sources have been reviewed and suggest an interpretation of the experimental excess and its observed shape in terms of a hadronic gas at a temperature of approximately $165 \mathrm{MeV} / \mathrm{c}$.

Assuming a first order phase transition, a two component structure observed in the single photon $p_{\perp}$-spectrum would allow for an extraction of the critical temperature $T_{c}$ and signal the formation of a QGP and its initial temperature by a hard component at larger values of $p_{\perp}$.

Prospects for single photon measurements at RHIC and LHC collider experiments seem rather encouraging. Recently, it has been pointed out by Shuryak and co-workers 38 and confirmed by parton-kinetics 27 that the thermal equilibration of gluons happens very fast leading to a 'hot-glue' scenario with very high gluon temperatures. Quark production on the other hand is much slower, so that a chemical non-equilibrium situation is expected. It is however found that the smaller quark number is more than compensated by the fact that they are embedded into the hotter glue. The predicted yields are thus larger than considered before.

In summary, the experimental detection of electromagnetic emission from the plasma is not going to be easy but the chances for the observation of the signals are realistic. The fact that they are the only direct signals from the quark-gluon plasma stage justifies a major experimental effort in this area.

\section{REFERENCES}

1. B. Petersson, Nucl. Phys. (Proc. Suppl.) B30 (1993) 66-80.

2. Proceedings to Quark-Matter Conference, Borlänge 1993, Nucl. Phys. A, 1993.

3. E. L. Feinberg, Nuovo Cimento 34 (1976) 391-412.

4. E. V. Shuryak, Phys. Lett. 78B (1978) 150-153.

5. K. Kajantie and H. I. Miettinen, Z. Phys. C9 (1981) 341-345.

6. F. Halzen and H. C. Liu, Phys. Rev. D25 (1982) 1842-1846.

7. L. D. McLerran and T. Toimela, Phys. Rev. D31 (1985) 545-563.

8. R. C. Hwa and K. Kajantie, Phys. Rev. D32 (1985) 1109-1118.

9. T. Ferbel and W. R. Molzon, Rev. Mod. Phys. 56 (1984) 181-221. 
10. J. F. Owens, Rev. Mod. Phys. 59 (1987) 465-503.

11. R. M. Turnbull, J. Phys. G14 (1988) 135-161.

12. L. Cormell and J. F. Owens, Phys. Rev. D22 (1980) 1609-1616.

13. M. Neubert, Z. Phys. C42 (1989) 231-242.

14. P. V. Ruuskanen, Nucl. Phys. A544 (1992) 169c-182c.

15. E. Braaten and R. D. Pisarski, Nucl. Phys. B337 (1990) 569-634 and Nucl. Phys. B339 (1990) 310-324.

16. R. Baier, et al., Z. Phys. C53 (1992) 433-438.

17. H. A. Weldon, Phys. Rev. D26 (1983) 2789-2796.

18. D. Bucher, Diploma thesis, University of Münster, 1993.

19. J. Kapusta, P. Lichard, and D. Seibert, Phys. Rev. D44 (1991) 2774-2788.

20. L. Xiong, E. Shuryak, and G. E. Brown, Phys. Rev. D46 (1992) 3798-3801.

21. M. Herrmann, B. L. Frieman, and W. Nörenberg, Z. Phys. A343 (1992) 119120.

22. G. Wolf, W. Cassing, and U. Mosel, Nucl. Phys. A552 (1993) 549-570.

23. P. Koch, Z. Phys. C57 (1993) 283-303.

24. K. Haglin and C. Gale, Preprint McGill/93-9, 1993.

25. J. Kapusta, L. McLerran, and D. K. Srivastava, Phys. Lett. B283 (1992) 145150.

26. S. Chakrabarty, et al., Phys. Rev. D46 (1992) 3802-3806.

27. K. Geiger and B. Müller, Nucl. Phys. B369 (1992) 600-654.

28. A. Dumitru, et al., Mod. Phys. Lett. A8 (1993) 1291.

29. H. Specht et al., CERES-Collaboration, Proceedings to this conference, 1993.

30. K.-H. Kampert et al., WA80-Collaboration, Proceedings to 2. International Conference on Physics and Astrophysics of the Quark-Gluon Plasma, Calcutta, January 19.-23., 1993, World Scientific, Singapore, 1993.

31. R. Santo et al., WA80-Collaboration, Proceedings to Quark-Matter Conference 1993, Borlänge, Nucl. Phys. A, 1993.

32. T.C. Awes et al., WA80-Collaboration, to be submitted to Phys. Lett. B, 1994.

33. R. Albrecht et al., WA80-Collaboration, Z. Phys. C51 (1991) 1-10.

34. T. Åkesson et al., NA34-Collaboration, Z. Phys. C46 (1990) 369-375.

35. G. Roche et al., DLS-Collaboration, Proceedings to this conference, 1993.

36. G. Clewing, Doctoral Thesis, University of Münster, 1993.

37. G. Hölker, Doctoral Thesis, University of Münster, 1993.

38. E. Shuryak, Phys. Rev. Lett. 22 (1992) 3270-3272 and

E. Shuryak and L. Xiong, Phys. Rev. Lett. 70 (1993) 2241-2244. 
